\title{
MS07-04 | The Neutron Structure of Leishmania Mexicana Triose Phosphate Isomerase With Transition State Mimics Reveals General Base Catalyst
}

Kelpsas, Vinardas (Lund University, Lund, SWE); Caldararu, Octav (Lund University, Lund, SWE); Kulkarni, Yashraj (Uppsala University, Uppsala, SWE); Wierenga, Rikkert (University of Oulu, Oulu); Ryde, Ulf (Lund University, Lund, SWE); Kamerlin, Lynn (Uppsala University, Uppsala, SWE); von Wachenfeldt, Claes (Lund University, Lund, SWE); Oksanen, Esko (European Spallation Source ESS ERIC, Lund, SWE)

Triose phosphate isomerase (TIM) is a key enzyme in glycolysis that catalyses the interconversion of glyceraldehyde-3-phosphate and dihydroxyacetone phosphate. This simple reaction involves the shuttling of protons mediated by protolysable sidechains. The catalytic power of TIM is believed to stem from the ability to deprotonate a carbon next to a carbonyl group, generating an enediolate intermediate. The enediolate intermediate is believed to be mimicked by the inhibitor 2-phosphoglocollate (PGA) and the following enediol intermediate by phosphoglycolohydroxamate (PGH) We have determined the neutron structure of Leishmania mexicana TIM with both inhibitors and performed joint neutron-X-ray refinement followed by quantum refinement. The structures clearly show that in the complex PGA the postulated general base E167 is protonated, while in the PGH complex it remains deprotonated. The deuteron is also clearly localised on E167, which suggests that the hydrogen bond is a double-well hydrogen bond instead of a low-barrier hydrogen bond. The full picture of active site protonation states allows us to simulate the deprotonation step of the reaction at the empirical valence bond level as well as snapshots with ab initio quantum chemistry, confirming our mechanistic explanation. 